\title{
The influence of treatment in alpine and moderate maritime climate on the composition of the skin microbiome in patients with difficult to treat atopic dermatitis
}

\author{
Minke M.F. van Mierlo' (D) | Joan E.E. Totté ${ }^{1}$ (D) | Karin B. Fieten ${ }^{2,3,4}$ (D) | \\ Tim J. van den Broek ${ }^{5}$ | Frank H.J. Schuren ${ }^{5}$ | Luba M. Pardo ${ }^{1}$ | Suzanne G.M.A. Pasmans ${ }^{1,2}$
}

${ }^{1}$ Department of Dermatology-Center of Paediatric Dermatology, Sophia Children's Hospital, Erasmus MC University Medical Center Rotterdam, Rotterdam, The Netherlands

${ }^{2}$ Department of Dermatology and Allergology, University Medical Center, Utrecht, The Netherlands

${ }^{3}$ Dutch Asthma Center Davos, Davos, Switzerland

${ }^{4}$ Swiss Institute of Allergy and Asthma Research (SIAF), University of Zürich, Davos, Switzerland

${ }^{5}$ Microbiology and Systems Biology, TNO Zeist, The Netherlands

\section{Correspondence}

Minke van Mierlo, Department of

Dermatology, Erasmus MC University

Medical Center Rotterdam, Dr.

Molewaterplein 40, 3015 GD Rotterdam,

The Netherlands.

Email:m.vanmierlo@erasmusmc.nl

Suzanne Pasmans, Department of Dermatology-Center of Paediatric Dermatology, Sophia Children's Hospital, Erasmus MC University Medical Center Rotterdam, Sp-1540, Wytemaweg 80, 3015 CN Rotterdam, The Netherlands.

Email: s.pasmans@erasmusmc.nl

Funding information

Micreos Human Health, the Netherlands; Merem Dutch Asthma Center Davos; European Allergy and Asthma Center Davos; patient support group "Vereniging Nederland Davos"

\begin{abstract}
Background: The skin microbiome, characterized by an overgrowth of Staphylococcus aureus, plays an important role in the pathogenesis of atopic dermatitis (AD). Multidisciplinary treatment in alpine climate is known for its positive effect on disease severity in children with $A D$ and can result in a different immune response compared with moderate maritime climate. However, the effect on the composition of the skin microbiome in $A D$ is unknown.

Objective: To determine the effect of treatment in alpine climate and moderate maritime climate on the microbiome for lesional and non-lesional skin in children with difficult to treat $A D$.

Results: Alpine climate treatment led to a significant change in the microbiota on lesional skin, whereas no significant change was found after moderate maritime climate. On both lesional and non-lesional skin, we observed a significant increase in Shannon diversity and a significant decrease in both Staphylococcus abundance and $S$ aureus load after alpine climate treatment. The decrease in $S$ aureus was significantly larger on lesional skin following alpine climate treatment compared with moderate maritime climate treatment. Staphylococcus epidermidis load was stable over time.

Conclusions and clinical relevance: Alpine climate treatment leads to significant changes in the composition of the skin microbiome in children with $A D$, mainly caused by a reduction in the Staphylococcus genus. This study shows new perspectives in the potential mode of action for therapies in AD.
\end{abstract}

\section{KEYWORDS}

atopic dermatitis, dermatology, microbiome, paediatrics, staphylococcus

\section{1 | INTRODUCTION}

Atopic dermatitis $(A D)$ is a chronic, relapsing skin disease with a prevalence of $10 \%-20 \%$ in West-European children. ${ }^{1} \mathrm{AD}$ is characterized by immunological changes, skin barrier dysfunction and 
microbiome is in constant interaction with the skin barrier and immune system, reinforcing the process of inflammation. ${ }^{4-6}$ The skin in $A D$ is characterized by an overgrowth of Staphylococcus aureus and analysis of the skin microbiome in different disease states of AD showed a correlation between the abundance of $S$ aureus and disease severity, with a higher load during disease flares. ${ }^{7}$ Also, a reduced diversity of other bacteria on the skin was found..$^{6-8}$

Atopic dermatitis treatment involves epidermal barrier repair using emollients, anti-inflammatory therapy using corticosteroids and trigger avoidance. In cases of severe (or infected) AD, systemic treatment and antimicrobial therapy is used. ${ }^{9,10}$ Alpine climate therapy has been used in patients with asthma and/or AD for decades. Alpine climate is characterized by lower exposure to allergens and pollution and an increased ultraviolet radiation (UV-R). ${ }^{11-13}$ Previous research, evaluating the effect of alpine climate treatment in patients with AD, showed improvement in disease activity and a reduced use of topical corticosteroids. ${ }^{13,14}$ The rationale of alpine climate therapy is mainly based on trigger avoidance and dampening the immune response. A recent study exploring the underlying immunological effects of alpine climate therapy found a significant reduction in blood eosinophils and an increase in circulating memory B cells, CD $8+T$ cells and Th2 cells which reflected a reduction in disease severity. ${ }^{15-17}$

It is known that climate factors could influence the skin microbiome. A study performed in healthy individuals within different humidity and temperature conditions showed an effect on the quantities of bacteria on the skin. ${ }^{18}$ Furthermore, UV-R can modulate the skin microbiome by causing direct microbial DNA damage and by affecting the immune system. ${ }^{19-22}$

Evaluation of alpine climate treatment has mainly focused on the immune system. The effect of alpine climate on the skin microbiome is still unclear. Identification of the skin microbiome and factors influencing the microbial composition might help in developing treatment strategies that improve disease severity by targeting the microbiome. Therefore, the aim of this study was to assess the effect of alpine climate treatment on the skin microbiome in children with difficult to treat $A D$ in a randomized controlled trial (RCT), comparing 6 weeks of alpine climate treatment with treatment in moderate maritime climate. ${ }^{23}$

\section{2 | METHODS}

\section{1 | Study design}

This study is incorporated in the DAVOS trial, a pragmatic RCT including children with difficult to treat AD. The trial is registered at Current Controlled Trials (ISRCTN88136485). The detailed study protocol and primary outcomes have been published previously. ${ }^{14,23}$ Briefly, Dutch children and adolescents were randomized to a six-week personalized integrative multidisciplinary (PIM) treatment in either a clinic in the Swiss alps at 1560 metres (intervention, alpine climate group) or an outpatient treatment programme in the Netherlands in moderate maritime climate (control, moderate maritime climate group). Patients were assessed before the start of treatment (time-point T0) and within 72 hours after the end of the six-week treatment (time-point T1). All study assessments were performed in the Netherlands. Study procedures were reviewed and approved by the Medical Ethics Committee of the University Medical Center Utrecht, the Netherlands (reference 09-192/K). This study involves secondary outcomes of this trial.

\section{2 | Participants}

Dutch children between 8 and 18 years old, with difficult to treat AD were eligible for participation in the study. We defined difficult to treat as use of at least a class three topical corticosteroid and not being able to step down, or current use of systemic immunosuppressive treatment, or repeated treatment with potent topical corticosteroids or systemic immunosuppressive treatment, or a history of use of systemic treatment, or a significant impact of $A D$ on the child's or the families quality of life, or seemingly unresponsive to conventional therapy according to current guidelines. ${ }^{14}$ All patients and if needed, their parents provided written informed consent. Demographic data were extracted from questionnaires and the electronic patient files. Microbiome samples were obtained prior to and after the end of the six-week treatment in the Wilhelmina Children's Hospital, the Netherlands.

\section{3 | Microbial samples}

Microbial samples were collected from the lesional and non-lesional skin. Samples taken from the lesional skin were preferably taken from the antecubital fold or the popliteal fold. Non-lesional skin samples were taken from the volar arm if possible. We used sterile cotton swabs soaked in sterile $\mathrm{NaCl} 0.9 \%$. Skin samples were collected by rubbing the skin for 30 seconds. All samples were stored at $-80^{\circ} \mathrm{C}$ until further processing.

\subsection{DNA isolation and qPCR}

For DNA isolation, phenol extraction and magnetic beads were used (Agowa mag Mini DNA isolation kit; LCG). First, $150 \mathrm{uL}$ from the sample was added to $350 \mathrm{uL}$ lysis buffer, $500 \mathrm{uL}$ phenol (Tris $\mathrm{pH} 8$ ) and $500 \mathrm{uL} \mathrm{0,1} \mathrm{mm} \mathrm{zirconium} \mathrm{beads.} \mathrm{This} \mathrm{mixture} \mathrm{was} \mathrm{me-}$ chanically disrupted with a beadbeater (Biospec products) twice for 2 minutes, followed by centrifuging for 10 minutes at 1690 RCF to separate the aqueous and phenolic phases. The aqueous phase was purified using AGOWA mag Mini DNA isolation kit. The bacterial DNA concentration measured after DNA extraction was performed using universal $16 \mathrm{~S}$ qPCR (16S-uni-I-F (5'-CGA AAG CGT GGG GAG CAA A-3'), 16S-uni-I-R (5'-GTT CGT ACT CCC CAG GCG G-3'), 16Suni-I MGB Taqman ${ }^{\circledR}$ probe (5'-ATT AGA TAC CCT GGT AGT CCA $-3^{\prime}$ ) with FAM ${ }^{\mathrm{TM}}$ (abel). Staphylococcus aureus and $S$ epidermidis load were quantified using multiplex quantitative (q)PCR with the following combination of primers and probes: 16S-S.aur-F1 (5'-GCG AAG AAC CTT ACC AAA TCT TG-3'), 16S-S.aur-R1 (5'-TGC ACC ACC TGT CAC TTT GTC-3'), 16S-S.aur MGB Taqman ${ }^{\circledR}$ probe (5'-CAT CCT TTG ACA ACT CT-3') with NED ${ }^{\text {TM }}$ label. 16S-S.epi-R1 (CAT GCA CCA CCT 
GTC ACT CTG T) and the 16S-S.epi MGB Taqman probe (CCT CTG ACC CCT CTA G) with VIC label. Forty cycles of qPCR were performed. The DNA concentration was reported as log10 transformed, femtogram per microlitre (fg/uL) in this paper. Detailed information about the DNA concentrations before log transformation are noted in Table S1.

\section{5 | $16 \mathrm{~S}$ rRNA sequencing and taxonomic classification}

Microbiome analysis was performed with massively sequencing of the $16 \mathrm{~S}$ rRNA gene using V4 hypervariable region on the Illumina MiSeq sequencer (Illumina). Barcoded DNA fragments spanning the V4 hypervariable region were amplified with a standardizing level of template DNA (1 ng). This was used to prevent over-amplification. Amplicons, generated using adapted primers F515 and R806 (using 30 PCR cycles), were bidirectionally sequenced using the MiSeq system. ${ }^{24}$ Samples containing insufficient amounts of DNA did not result in usable sequence data and were therefore omitted. Pre-processing and classification of sequences were performed using the Mothur V.1.31.1 software platform. To assign taxonomic names, the Ribosomal Database Project (RDP) Classifier was used. ${ }^{25}$ Technical performance was checked by using standardized mock communities. Negative control samples of the lysis buffer did not show signs of contamination. A genus table with raw read counts was generated for downstream analysis.

\subsection{Statistical analysis}

Our statistical analysis was performed in patients with available data at both time-points per outcome. Shannon diversity index was calculated at genus level on non-subsampled unfiltered data. For further analysis of the microbiome, we used non-subsampled genus tables and excluded genera with a relative abundance lower than 0.0001. Prior to ordination analysis, the filtered genus tables were square-root transformed with subsequent application of Wisconsin double standardization. To visualize bacterial community compositions, Bray-Curtis distance-based multidimensional scaling (MDS) was used. Permutational multivariable analysis of variance (PERMANOVA) was used to determine significant changes in microbiota. To assess whether the change in microbiota was significantly different between both treatment groups, we used the covariates "time-point" (TO or T1) and "treatment group" (alpine climate or moderate maritime climate) as interaction terms in this model. If any statistically significant difference was detected, we obtained PERMANOVA coefficients to determine which genera contributed most to this change. ${ }^{26}$

To detect changes in relative abundance within the 10 most abundant genera over time, we performed univariate analysis using a negative binomial generalized linear model. ${ }^{27}$ In-depth analysis was performed on $S$ aureus and $S$ epidermidis abundance by comparing $\log 10$ transformed concentrations (fg/uL). Undetectable DNA concentrations were noted as equal to zero and referred to as negative. A linear mixed-effect model with post hoc analysis was used to assess the changes in $S$ aureus and $S$ epidermidis. The differences between both treatment groups were assessed by calculating the interaction between covariates "time-point:treatment group". This statistical model was also applied to Shannon diversity index.

Statistical analysis was performed in SPSS (version 21) and R software (version 3.5.1). linear mixed-effect models were performed using "Ime" and "Ime4" package. Post hoc analysis was performed with "multcomp" package and corrected for multiple testing. ${ }^{28,29} \mathrm{We}$ used the packages "ape" and "vegan" for MDS and PERMANOVA, respectively. ${ }^{30}$ In this model, we accounted for repeated measurements using the "strata" argument. The changes in relative abundance for the 10 most abundant genera were analysed using package "DESeq2". 27 "ggplot 2" was used for visualization. ${ }^{31}$ A $P$-value of $\leq .05$ was considered statistically significant.

\section{3 | RESULTS}

\section{1 | Study subjects}

A total of 84 patients were randomized of whom 79 patients started the intervention. Two patients from the moderate maritime climate group did not complete intervention and sequencing data and qPCR data were missing for two other patients in this group. This resulted in 75 patients in our study: 38 patients in the alpine climate group and 37 patients in the moderate maritime climate group (Figure 1). Demographic data, comorbidities and disease severity were not significantly different between both groups at the start of intervention (Table 1). The median severity score at T0 was 39.0 (IQR 18.7-59.3) for the alpine climate group and 40.8 (IQR 22.2-52.8) for the moderate maritime climate group.

\section{2 | Sample characteristics}

Lesional and non-lesional skin samples were collected from all 75 patients in this study at both time-points and were analysed using qPCR techniques. Sufficient amount of DNA to perform 16S rRNA sequencing was available at both time-points in 49 patients for lesional skin and 45 patients for non-lesional skin (Figure S1). Patient characteristics of missing data did not differ from the study group. A total of 1603092 sequences (median 9600; IQR 3516-26420) were obtained from the 98 lesional samples and 1949477 sequences (median 28 780; IQR 6007-347885) from the 90 non-lesional samples. All sequences belonged to 603 genera. After filtering, 213 genera remained. The 10 most abundant genera on lesional and non-lesional skin, before and after both treatment regimens, are shown in Figure S2. Staphylococcus was predominant in all groups followed by the Corynebacterium genus and Streptococcus genus.

\subsection{The effect of alpine climate treatment on the microbial composition}

We visualized the microbiota separated for lesional and non-lesional skin (Figure 2, Figure S3). Before the start of treatment, the microbial 


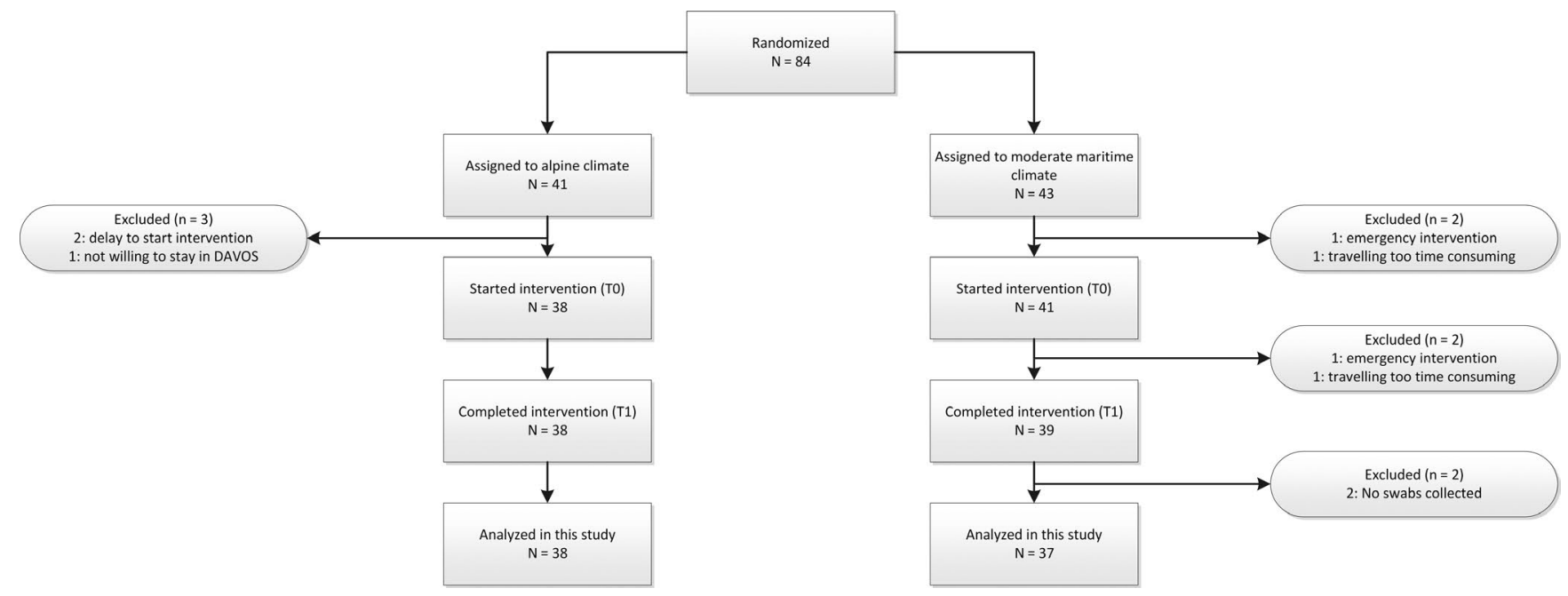

FIGURE 1 Flowchart of study participants

composition did not differ significantly between both treatment groups (PERMANOVA: lesional skin: $R^{2}=.025, P=.25$, non-lesional skin: $R^{2}=.025, P=.22$ ). After 6 weeks of alpine climate treatment, a significant shift in microbiota was observed on lesional skin (PERMANOVA: $R^{2}=.035, P=.01$ ), whereas no significant change occurred after treatment in moderate maritime climate (PERMANOVA: $\left.R^{2}=.011, P=.81\right)$. The observed change on lesional skin after alpine climate was mainly driven by Staphylococcus genus with a coefficient which was 2.7 times larger than for other genera (Figure S4). The interaction "time-point:treatment group" was not significant, indicating that the change in microbiota was not significantly different affected by treatment protocol $(P=$.19). The microbiota on non-lesional skin did not change significantly following either treatment (Figure S3).

Compared with baseline, Shannon diversity index was significantly increased after alpine climate treatment on both lesional and non-lesional skin $(P<.01$ and $P=.02$ respectively). This could not be observed after moderate maritime climate $(P=.26$ and $P=.70$, respectively). Moreover, the change in Shannon index was not significantly different between both treatment groups (lesional skin $P=.26$ and non-lesional skin $P=.07$; Figure 3 ).

\subsection{Effect of alpine climate treatment on the abundance of the 10 most abundant genera}

The Staphylococcus genus showed a significant reduction in the group treated in alpine climate on both lesional and non-lesional skin (both $P<.01)$. In the moderate maritime climate group, a significant reduction in Staphylococcus genus was found on lesional skin $(P<.01)$. The other 10 most abundant genera, as noted in Figure S2, were not significantly affected by both treatment regimens (Figure S5).

\subsection{Effect of alpine climate treatment on species within the Staphylococcus genus}

To get more insight in the Staphylococcus genus, additional qPCR was performed to identify $S$ aureus and $S$ epidermidis. For all participants,
qPCR data was available and included in our analysis $(n=75)$. In the total study population, 57 (76.0\%) patients were positive for $S$ aureus on lesional skin at T0 (alpine climate group: $\mathrm{n}=29$ [76.3\%] and moderate maritime climate group: $n=28$ [75.7\%], Table 1). At T1, 40 (53.3\%) patients remained positive on lesional skin (alpine climate group: $n=16$ [42.1\%] and moderate maritime climate group: $n=24$ [64.9\%]). These percentages were lower on non-lesional skin (TO: $n=19$ [50.0\%] and $\mathrm{n}=18$ [48.6\%] for alpine climate and moderate maritime climate, respectively, T1: $\mathrm{n}=10$ [26.3\%] and $\mathrm{n}=14$ [37.8\%], respectively). After 6 weeks of treatment, the decrease in $S$ aureus load on lesional skin was significantly different in patients treated in alpine climate compared with moderate maritime climate $(P=.02$; Figure 4). After alpine climate treatment $S$ aureus reduced from a median of $2.6 \mathrm{fg} / \mathrm{uL} \log 10$ (IQR 0.1-3.4) at T0 to $0.0 \mathrm{fg} / \mathrm{uL} \log 10$ (IQR 0.0-0.9) at T1 ( $>$ < .01), due to the proportion of patients with undetectable $S$ aureus concentration at T1. In the moderate maritime climate group, $S$ aureus decreased from a median of $2.0 \mathrm{fg} / \mathrm{uL} \log 10$ (IQR 0.3-3.0) to $1.1 \mathrm{fg} / \mathrm{uL} \log 10$ (IQR $0.0-2.7 ; P=.11$ ). On non-lesional skin, a significant drop in $S$ aureus load was observed after treatment in alpine climate with a median of $0.4 \mathrm{fg} / \mathrm{uL} \log 10$ (IQR 0.0-2.1) at T0 and $0.0 \mathrm{fg} / \mathrm{uL} \log 10$ (IQR 0.0-1.0) at T1 $(P<.01$; Figure S6). Staphylococcus epidermidis was positive in 71 (94.7\%) patients in the total study group on both lesional and non-lesional skin at T0, compared with 72 (96\%) and 74 (98.7\%) for lesional and non-lesional skin at T1 (Table 1). Staphylococcus epidermidis load was not affected by either treatment protocol (Figure 4 and Figure S6).

\section{4 | DISCUSSION}

This study showed that alpine climate treatment affects the microbiome on both lesional and non-lesional skin in children with difficult to treat $A D$. We found a significant change in the overall skin microbiome on lesional skin after 6 weeks of alpine climate treatment, whereas no significant change was observed after moderate maritime climate treatment. Moreover, a significant change was observed on both lesional and non-lesional skin in Shannon diversity 
TAB LE 1 Baseline characteristics

\begin{tabular}{|c|c|c|}
\hline & $\begin{array}{l}\text { Alpine climate group } \\
(\mathrm{n}=38)\end{array}$ & $\begin{array}{l}\text { Moderate } \\
\text { maritime climate } \\
\text { group }(n=37)\end{array}$ \\
\hline Sex (female), n(\%) & $17(44.7)$ & $19(51.4)$ \\
\hline Age, mean \pm SD & $13.1 \pm 2.5$ & $12.8 \pm 2.4$ \\
\hline $\begin{array}{l}\text { Age of AD onset } \\
<6 \mathrm{mo}, \mathrm{n}(\%)\end{array}$ & $6(15.8)$ & $5(13.5)$ \\
\hline Asthma ${ }^{a}, \mathrm{n}(\%)$ & $25(65.8)$ & $29(78.4)$ \\
\hline Rhinitis $^{\mathrm{b}}, \mathrm{n}(\%)$ & $34(89.5)$ & $32(86.5)$ \\
\hline Food allergy ${ }^{c}, \mathrm{n}(\%)$ & $26(68.4)$ & $26(70.3)$ \\
\hline $\begin{array}{l}\text { SA-EASI, median } \\
\text { (IQR) }\end{array}$ & $39.0(18.7-59.3)$ & $40.8(22.2-52.8)$ \\
\hline \multicolumn{3}{|c|}{ Topical corticosteroids ${ }^{d}, n(\%)$} \\
\hline None & $1(2.6)$ & $1(2.7)$ \\
\hline Moderate & $1(2.6)$ & $3(8.1)$ \\
\hline Potent & $35(92.1)$ & $30(81.1)$ \\
\hline Very potent & $1(2.6)$ & $3(8.1)$ \\
\hline \multicolumn{3}{|c|}{ Systemic medication, n(\%) } \\
\hline Prednisone & $3(7.9)$ & - \\
\hline Cyclosporine & $1(2.6)$ & $4(10.8)$ \\
\hline Oral antibiotics, $n(\%)$ & - & $1(2.7)$ \\
\hline \multicolumn{3}{|c|}{ Positive for $\mathrm{S}$ aureus, $\mathrm{n}(\%)^{\mathrm{e}}$} \\
\hline Lesional skin & $29(76.3)$ & $28(75.7)$ \\
\hline Non-lesional skin & $19(50.0)$ & $18(48.6)$ \\
\hline \multicolumn{3}{|c|}{ Positive for S epidermidis, $\mathrm{n}(\%)^{\mathrm{e}}$} \\
\hline Lesional skin & $35(92.1)$ & $36(97.3)$ \\
\hline Non-lesional skin & $36(94.7)$ & $35(94.6)$ \\
\hline
\end{tabular}

Abbreviations: AD, atopic dermatitis; SA-EASI, Self-Administered Eczema Area and Severity Index; SD, standard deviation.

${ }^{a}$ Asthma was diagnosed based on spirometry reversibility testing and Methacholine Challenge Test.

${ }^{\mathrm{b}}$ Rhinitis was diagnosed based on assessment by a paediatrician.

${ }^{c}$ Food allergy was defined as a positive double-blind placebo-controlled food challenge (DBPCFC) or convincing clinical history (a reported Type I allergic reaction with acute symptoms within $2 \mathrm{~h}$ after ingestion of the food) in combination with sensitization to the specific food allergen.

dUK potency system used.

'Determined using qPCR methods.

index, Staphylococcus abundance and $S$ aureus load in particular. The reduction in $S$ aureus load was significantly larger when compared to moderate maritime climate. For the group treated in moderate maritime climate, only a significant reduction on lesional skin for the Staphylococcus genus was found.

This is the first study describing the effect of alpine climate treatment on the skin microbiome in patients with AD. Alpine climate offers favourable features for patients with $A D$, including lower exposure to allergens and pollution and an increased UV-R. ${ }^{11,12}$ Moreover, children treated in alpine climate were separated from their parents and intensively monitored by the multidisciplinary treatment team. This treatment setting in alpine climate has beneficial effects on disease severity and was shown to affect the immune
Lesional skin

Microbiome composition; Bray-Curtis distance

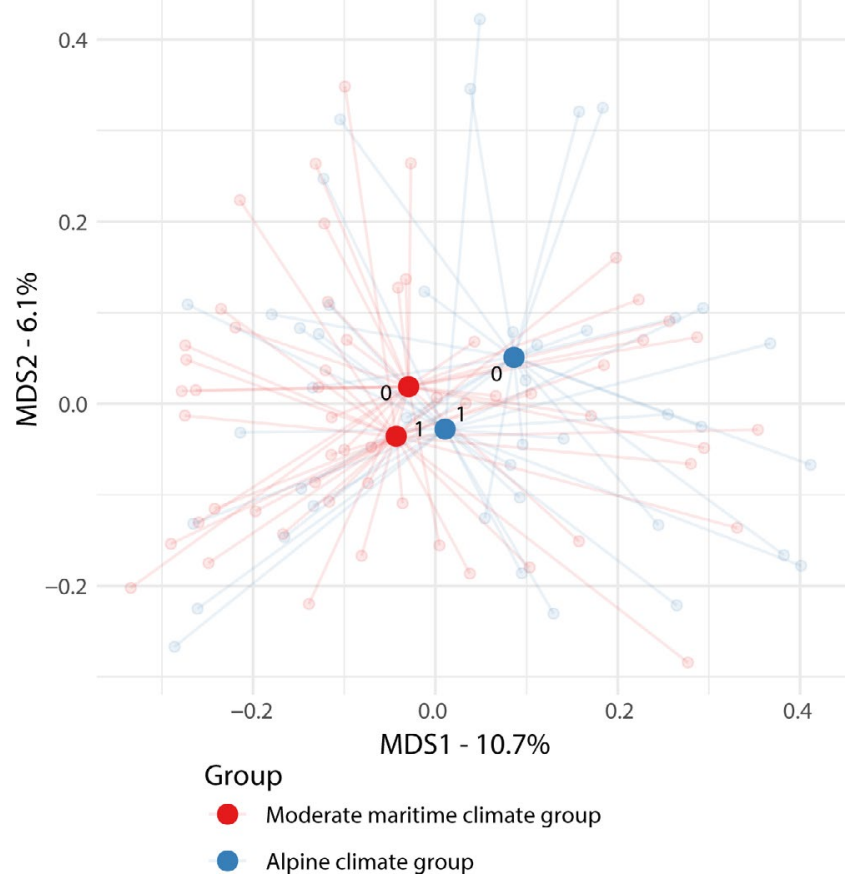

FIGURE 2 Bray-Curtis distance-based multi-dimensional scaling plot showing the microbiota of the lesional skin samples before and after the six-week intervention period in alpine climate and moderate maritime climate. Statistical analysis was performed on 21 patients in the alpine climate treatment group and 28 patients in moderate maritime climate group. For ordination analysis, genus tables were standardized using square-root transformation with subsequent application of Wisconsin double standardization. The change in microbiota was significant for patients treated in alpine climate (PERMANOVA: $R^{2}=.035, P=.01$ ). No significant change was observed after moderate maritime climate treatment (PERMANOVA: $R^{2}=.011, P=.81$ ). MDS 1: dimension 1 , representing $10.7 \%$ of total variation. MDS 2: dimension 2 , representing $6.1 \%$ of total variation. T0: before the start of the intervention period. T1: after 6 wk of treatment

response (blood eosinophils, memory B cells, CD8+ T cells and Th2 cells). ${ }^{15,19,32}$ Besides effects on the immune system, studies also proposed that geographical variability, with variation in UV-R, can influence the skin barrier and the microbiome. ${ }^{2,33}$ Although this study shows a change in the skin microbiome after alpine climate treatment, we cannot prove that the observed effect is directly caused by the alpine climate or through the effect on the immune system or treatment setting.

After alpine climate treatment, we found a significant increase in Shannon diversity index and a significant reduction in the quantity of $S$ aureus on both lesional and non-lesional skin. Moreover, the decrease in $S$ aureus load on lesional skin was significantly different from the maritime climate group $(P=.02)$. In a previous paper, describing the effectiveness of alpine climate treatment in this study population, a significantly larger decrease in disease severity was observed following alpine climate treatment than maritime climate 


\section{Shannon diversity}

(A) Lesional skin
(B) Non-lesional skin

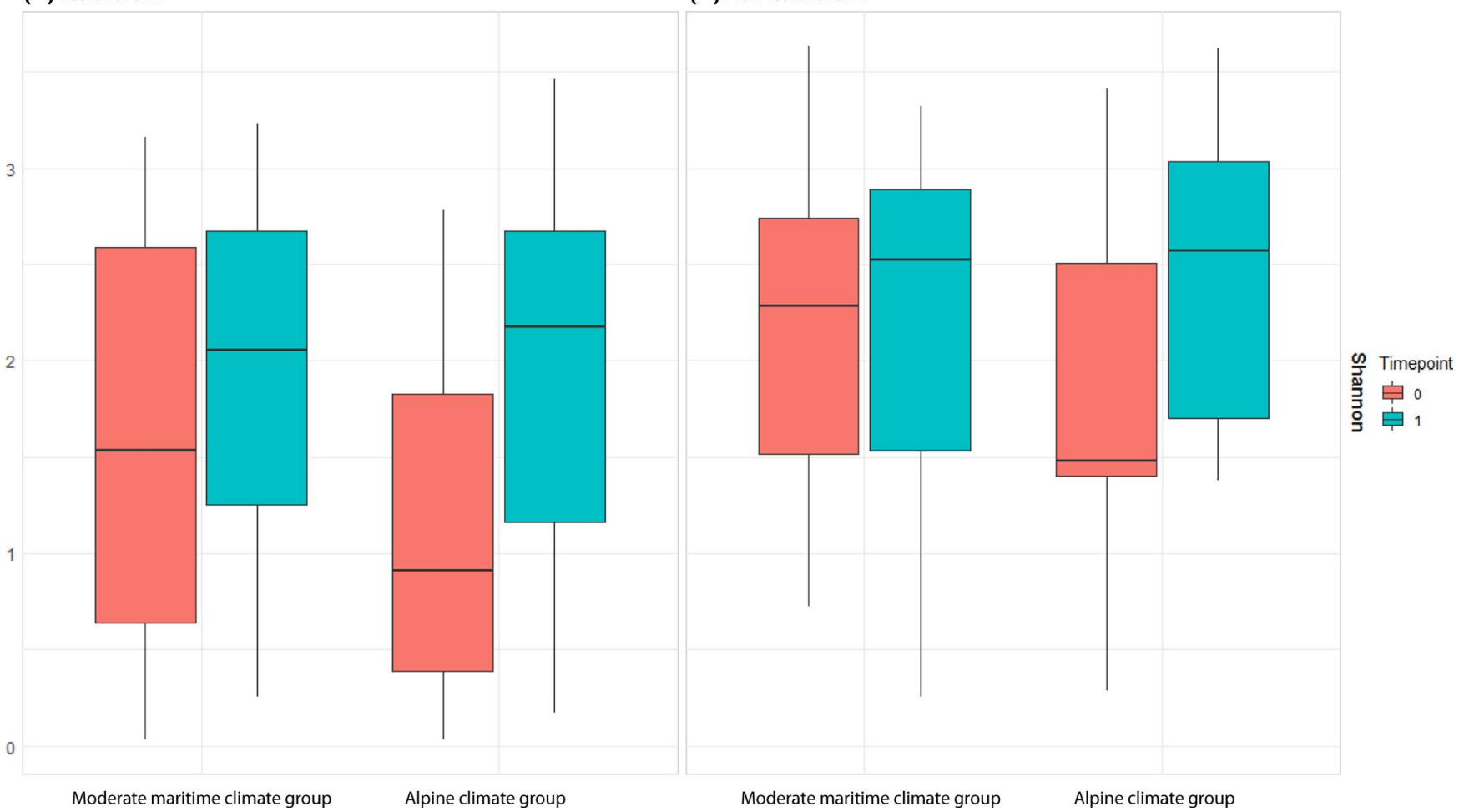

FIGURE 3 Shannon diversity index of skin samples before and after the six-week intervention period in alpine climate and moderate maritime climate. Shannon diversity index represents the number of different genera (richness) and how even they are distributed (evenness). The boxes represent the 25 th percentile, median and 75 th percentile. Statistical analysis was performed using a linear mixed-effect model with post hoc analysis. Time-point 0: before the start of the intervention period. Time-point 1: after 6 wk of treatment. A, Lesional skin samples. Statistical analysis was performed on 21 patients in the alpine climate treatment group and 28 patients in moderate maritime climate group. Compared with baseline, significant differences in Shannon diversity index were found after treatment in alpine climate $(P<.01)$. No significant change was observed after moderate maritime climate $(P=.09)$ or between both treatment groups $(P=.26)$. B, Non-lesional skin samples. Statistical analysis was performed on 19 patients in the alpine climate treatment group and 26 patients in moderate maritime climate group. Compared with baseline, significant differences in Shannon diversity index were found after treatment in alpine climate $(P=.02)$. No significant change was observed after moderate maritime climate $(P=.70)$ or between both treatment groups $(P=.07)$

treatment $(P<.01)$. The SA-EASI score decreased from a median of 39.0 (IQR 18.7-59.3) to 2.6 (IQR 0.3-6.2) and 40.8 (IQR 22.2-52.8) to 12.0 (IQR 3.6-22.1) after six-week treatment in alpine climate and moderate maritime climate, respectively. ${ }^{14}$ Literature shows a positive correlation between the abundance of Staphylococcus, in particular $S$ aureus, and disease severity in patients with AD. ${ }^{6-8,34} \mathrm{~A}$ decrease in disease severity also leads to higher bacterial diversity. ${ }^{7}$ It is likely that our results are affected by the differences in disease severity. However, it is unknown whether the $S$ aureus abundance is a result or a cause of changes in disease severity. More studies with frequent sampling around flares are needed to answer this question.

Compared with lesional skin, we were not able to detect a change in the microbiota on non-lesional skin after alpine climate treatment. However, the changes in Shannon diversity index, Staphylococcus abundance and $S$ aureus after alpine climate treatment were significant for both lesional and non-lesional skin. Non-lesional skin in AD is known to differ from both lesional AD skin and healthy skin. ${ }^{35,36}$ This can be explained by the impaired skin barrier in patients with $A D$, which also affects non-lesional skin and makes it more susceptible to penetration of allergens and bacteria than healthy skin. ${ }^{37} \mathrm{~A}$ previous study investigating the effect of topical corticosteroids and bleach baths in patients with $A D$ also showed a significant change in microbial composition after treatment on lesional skin, but not for non-lesional skin. ${ }^{8}$ This is in line with our findings. A possible explanation can be the higher diversity and lower Staphylococcus abundance, compared with lesional skin, making changes more subtle. Another explanation can be the lesser impact of disease severity and thus inflammation on this skin. Non-lesional skin might tell us more about the effect of climate than lesional skin which is more subject to the secondary effects of inflammation.

In this study, both lesional and non-lesional skin in AD patients were dominated by the Staphylococcus genus. These results support previous literature describing an excess of Staphylococcus, and more specific of $S$ aureus, in the skin microbiome of patients with AD. ${ }^{3,7,36}$ Other abundant genera included Streptococcus (known to be more present in children with $\mathrm{AD}$ ) and Corynebacterium (common in healthy skin microbiome). ${ }^{3,35,38}$ Looking in more depth at the Staphylococcus genus, the prevalence of $S$ aureus at the start of intervention was $76.0 \%$ and $49.3 \%$ for lesional and non-lesional skin, respectively. These percentages are slightly higher than described in 


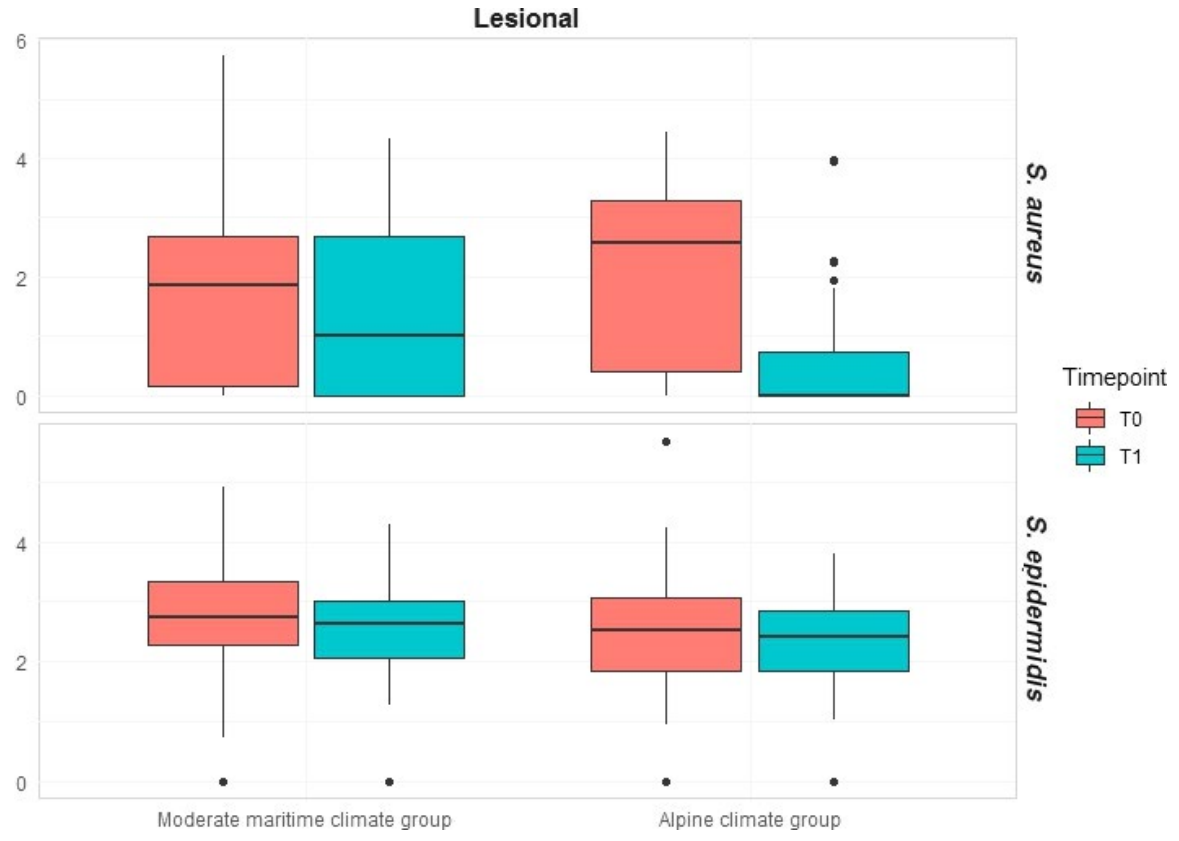

FIG URE 4 Quantitative PCR results of the lesional skin samples for Staphylococcus aureus and S epidermidis before and after the six-week intervention period in alpine climate and moderate maritime climate. The results for $S$ aureus and $S$ epidermidis load are shown using log 10 transformed data. Statistical analysis was performed on 38 patients in the alpine climate treatment group and 37 patients in moderate maritime climate group. A significant difference in $S$ aureus load was found after alpine climate treatment $(P<.01)$. Staphylococcus epidermidis was stable during the treatment period. The decrease in $S$ aureus load was significantly larger after alpine climate compared with moderate maritime climate $(P=.02)$. The boxes represent the 25 th percentile, median and 75 th percentile. Dots represent individual samples. Statistical analysis was performed using a linear mixed-effect model with post hoc analysis. Time-point 0: before the start of the intervention period. Time-point 1: after six weeks of treatment

a recent meta-analysis on this subject and might be explained by our inclusion criteria, selecting patients with moderate to severe AD. ${ }^{39}$ Although patients showed a significant reduction in disease severity in this study, a large proportion remained positive for $S$ aureus on the skin (lesional T0: 76,0\%, T1: 53,3\% and non-lesional T0: 49,3\%, T1: $32,0 \%)$. These results suggest that AD symptoms are not only associated with the presence or absence of $S$ aureus, but more importantly with the total $S$ aureus load on the skin.

We did not find a significant change in $S$ epidermidis load in our study. The role of $S$ epidermidis in the pathogenesis of $A D$ is still unclear and literature on this subject is conflicting. ${ }^{7,40,41}$ Due to the inhibitory effect of $S$ epidermidis on $S$ aureus, by the production of bacteriocins, serine protease Esp and phenol-soluble modulins, some correlation may be expected. ${ }^{42-44}$ In a previous study, an increase in $S$ epidermidis was found during disease flare in patients with $A D{ }^{7}$ In our study, the quantities of $S$ epidermidis were stable over time despite a drop in $S$ aureus load and severity following treatment. It is possible that $S$ epidermidis is elevated in the acute stadium (flares) as a compensatory mechanism to control $S$ aureus, but in a chronic stadium these levels normalized.

This study has a pragmatic design, which makes it hard to assess what contributed most to the observed outcomes. Characteristic of the alpine climate group, besides the unique aspects of this climate, was the supervision leading to optimal treatment compliance. It might be that due to supervision, the application of topical corticosteroids was more adequately and frequent in this group and could have affected disease severity and the skin microbiome. Moreover, this study describes secondary outcomes of this trial and medication use was not applied as exclusion criteria. Previous studies show an effect of medication use on the skin microbiome. However, it was not possible to discontinue medication use in this group of patients with difficult to treat, moderate to severe disease. ${ }^{8,45}$ During this study, the use of medication was carefully monitored and showed no significant differences between both treatment groups. Results for patients using systemic medication at TO did not deviate from the rest of the study population (data not shown). Moreover, the proportion of patients using topical and/or systemic medication during the intervention was stable (Table S2).

A limitation of this study was the use of the V4 hypervariable region for sequencing. With this variable region it is not possible to properly detect the Propionibacterium or to classify the Staphylococci at species level. To overcome this problem for the Staphylococci, we determined $S$ aureus and S epidermidis abundance with qPCR methods. Since the body sites which were mainly sampled for this study are usually low or devoid of Propionibacterium this should only have had a minor to negligible effect on the data presented here. ${ }^{2}$

This study encourages to perform explanatory studies with a similar treatment setting in both climates to confirm the effect of climate conditions alone on the skin microbiome. Including samples from the skin of healthy subjects in future studies might give us more 
information about the differences in the skin microbiome between $A D$ and controls and assess if a decrease in disease severity (which was observed at T1) leads to a microbiome more comparable to healthy subjects. Furthermore, it would be interesting to assess if residents of moderate maritime climate and alpine climate have distinct microbiota.

In conclusion, 6 weeks of treatment in the alpine climate, affects the skin microbiome in children with difficult to treat AD. In contrast to moderate maritime climate, alpine climate treatment caused a significant change in the microbiota on lesional skin. In addition, we found a significant increase in Shannon diversity index and a significant decrease in abundance of the Staphylococcus genus and $S$ aureus load on both lesional and non-lesional skin. This study shows new perspectives in the potential mode of action for therapies in patients with $A D$ and encourages further investigation of skin microbiome modulating therapies.

\section{ACKNOWLEDGEMENT}

The authors are very grateful to the children and their parents for participating in the study and to J. Beutler for assistance in collection of the samples. We thank the Dutch patient support group VMCE for endorsing the study. We want to acknowledge all dermatologists for enabling patients to participate in our study by referring them to our hospital. This study was supported by unrestricted grants from the European Allergy and Asthma Center Davos, the Merem Dutch Asthma Center Davos, the patient support group "Vereniging Nederland Davos" and Micreos Human Health, the Netherlands.

\section{CONFLICT OF INTEREST}

This study was supported by unrestricted grants from the European Allergy and Asthma Center Davos, the Merem Dutch Asthma Center Davos, the patient support group "Vereniging Nederland Davos" and Micreos Human Health, The Netherlands. These parties were not involved in analysis of the data or the writing of this paper.

\section{DATA AVAILABILITY STATEMENT}

The data that support the findings of this study are available from the corresponding author upon reasonable request.

\section{ORCID}

Minke van Mierlo iD https://orcid.org/0000-0001-5397-7382

Joan Totté (iD https://orcid.org/0000-0002-3231-4720

Karin Berthine Fieten iD https://orcid.org/0000-0003-3790-7581

\section{REFERENCES}

1. Odhiambo JA, Williams HC, Clayton TO, Robertson CF, Asher MI, Group IPTS. Global variations in prevalence of eczema symptoms in children from ISAAC phase three. J Allergy Clin Immunol. 2009;124(6):1251-1258.e23.
2. Grice EA, Segre JA. The skin microbiome. Nat Rev Microbiol. 2011;9(4):244-253.

3. Bjerre RD, Bandier J, Skov L, Engstrand L, Johansen JD. The role of the skin microbiome in atopic dermatitis: a systematic review. $\mathrm{Br} J$ Dermatol. 2017;177(5):1272-1278.

4. Geoghegan JA, Irvine AD, Foster TJ. Staphylococcus aureus and atopic dermatitis: a complex and evolving relationship. Trends Microbiol. 2018;26(6):484-497.

5. Travers JB. Toxic interaction between Th2 cytokines and Staphylococcus aureus in atopic dermatitis. J Invest Dermatol. 2014;134(8):2069-2071.

6. Clausen ML, Agner T, Lilje B, Edslev SM, Johannesen TB, Andersen PS. Association of disease severity with skin microbiome and filaggrin gene mutations in adult atopic dermatitis. JAMA Dermatol. 2018;154(3):293-300

7. Kong $\mathrm{HH}, \mathrm{Oh} J$, Deming $\mathrm{C}$, et al. Temporal shifts in the skin microbiome associated with disease flares and treatment in children with atopic dermatitis. Genome Res. 2012;22(5):850-859.

8. Gonzalez ME, Schaffer JV, Orlow SJ, et al. Cutaneous microbiome effects of fluticasone propionate cream and adjunctive bleach baths in childhood atopic dermatitis. J Am Acad Dermatol. 2016;75(3): 481-493.e8.

9. Ring J, Alomar A, Bieber T, et al. Guidelines for treatment of atopic eczema (atopic dermatitis) part I. J Eur Acad Dermatol Venereol. 2012;26(8):1045-1060

10. Ring J, Alomar A, Bieber T, et al. Guidelines for treatment of atopic eczema (atopic dermatitis) part II. J Eur Acad Dermatol Venereol. 2012;26(9):1176-1193.

11. Vocks E. Climatotherapy in atopic eczema. In: Ring J, Ruzicka T, edn. Handbook of atopic eczema. Berlin, Heidelberg: Springer-Verlag, Springer; 2006:507-523.

12. Rijssenbeek-Nouwens LH, Bel EH. High-altitude treatment: a therapeutic option for patients with severe, refractory asthma? Clin Exp Allergy. 2011;41(6):775-782.

13. Fieten KB, Weststrate AC, van Zuuren EJ, Bruijnzeel-Koomen CA Pasmans SG. Alpine climate treatment of atopic dermatitis: a systematic review. Allergy. 2015;70(1):12-25.

14. Fieten K, Schappin R, Zijlstra W, et al. Effectiveness of alpine climate treatment for children with difficult to treat atopic dermatitis: results of a pragmatic randomized controlled trial (DAVOS trial). Clin Exp Allergy. 2018;48(2):186-195.

15. Heeringa JJ, Fieten KB, Bruins FM, et al. Treatment for moderate to severe atopic dermatitis in alpine and moderate maritime climates differentially affects helper T cells and memory B cells in children. Clin Exp Allergy. 2018;48(6):679-690.

16. Simon D. The effects of high altitude climate therapy. Phys Med Rehab Kuror. 2001:11:104-109.

17. Triebskorn A, Gloor M, Borelli S. Hydration of the horny layer and barrier function in atopic dermatitis before and after therapy in high-altitude climate. Z Hautkr. 1991;66:145-147.

18. McBride ME, Duncan WC, Knox JM. The environment and the microbial ecology of human skin. Appl Environ Microbiol. 1977;33(3):603-608.

19. Faergemann J, Larko O. The effect of UV-light on human skin microorganisms. Acta Derm Venereol. 1987;67(1):69-72.

20. Patra V, Byrne SN, Wolf P. The skin microbiome: is it affected by UV-induced immune suppression? Front Microbiol. 2016;7:1235

21. Thyssen JP, Zirwas MJ, Elias PM. Potential role of reduced environmental UV exposure as a driver of the current epidemic of atopic dermatitis. J Allergy Clin Immunol. 2015;136(5):1163-1169.

22. Wang $\mathrm{Y}$, Zhu W, Shu M, et al. The response of human skin commensal bacteria as a reflection of UV radiation: UV-B decreases porphyrin production. PLoS One. 2012;7(10):e47798.

23. Fieten KB, Zijlstra WT, van Os-Medendorp H, et al. Comparing high altitude treatment with current best care in Dutch children with 
moderate to severe atopic dermatitis (and asthma): study protocol for a pragmatic randomized controlled trial (DAVOS trial). Trials. 2014;15:94.

24. Caporaso JG, Lauber CL, Walters WA, et al. Ultra-high-throughput microbial community analysis on the Illumina HiSeq and MiSeq platforms. Isme J. 2012;6(8):1621-1624.

25. Wang Q, Garrity GM, Tiedje JM, Cole JR. Naive bayesian classifier for rapid assignment of rRNA sequences into the new bacterial taxonomy. Appl Environ Microbiol. 2007;73(16):5261-5267.

26. González I, Déjean S, Martin PGP, Baccini A. CCA: An R Package to Extend Canonical Correlation Analysis. Journal of Statistical Software, 2008;23(12):14

27. Love MI, Huber W, Anders S. Moderated estimation of fold change and dispersion for RNA-seq data with DESeq2. Genome Biol. 2014;15(12):550.

28. Harrison XA, Donaldson L, Correa-Cano ME, et al. A brief introduction to mixed effects modelling and multi-model inference in ecology. PeerJ. 2018;6:e4794.

29. Benjamini $Y$, Hochberg Y. Controlling the false discovery rate: a practical and powerful approach to multiple testing. J Roy Stat Soc: Ser B (Methodol). 1995;57(1):289-300.

30. Oksanen J, Blanchet FG, Friendly M, Kindt R, Legendre P, McGlinn $\mathrm{D}$, et al. vegan: Community Ecology Package. Ordination methods, diversity analysis and other functions for community and vegetation ecologists. Version 2.5-1. https://CRAN.R-project.org/packa ge=vegan 2018

31. Wickham H. ggplot2: elegant graphics for data analysis. New York, NY: Springer-Verlag; 2009.

32. Hamann CR, Andersen $Y$, Engebretsen KA, et al. The effects of season and weather on healthcare utilization among patients with atopic dermatitis. J Eur Acad Dermatol Venereol. 2018;32(10):1745-1753.

33. Engebretsen KA, Johansen JD, Kezic S, Linneberg A, Thyssen JP. The effect of environmental humidity and temperature on skin barrier function and dermatitis. J Eur Acad Dermatol Venereol. 2016;30(2):223-249.

34. Gong JQ, Lin L, Lin T, et al. Skin colonization by Staphylococcus aureus in patients with eczema and atopic dermatitis and relevant combined topical therapy: a double-blind multicentre randomized controlled trial. Br J Dermatol. 2006;155(4):680-687.

35. Shi B, Bangayan NJ, Curd E, et al. The skin microbiome is different in pediatric versus adult atopic dermatitis. J Allergy Clin Immunol. 2016;138(4):1233-1236.

36. Seite S, Flores GE, Henley JB, et al. Microbiome of affected and unaffected skin of patients with atopic dermatitis before and after emollient treatment. J Drugs Dermatol. 2014;13(11): $1365-1372$.
37. Irvine $A D$, McLean $\mathrm{WH}$, Leung DY. Filaggrin mutations associated with skin and allergic diseases. N Engl J Med. 2011;365(14): 1315-1327.

38. Grice EA, Kong HH, Conlan S, et al. Topographical and temporal diversity of the human skin microbiome. Science. 2009;324(5931): 1190-1192.

39. Totte JE, van der Feltz WT, Hennekam M, van Belkum A, van Zuuren EJ, Pasmans SG. Prevalence and odds of Staphylococcus aureus carriage in atopic dermatitis: a systematic review and meta-analysis. $\mathrm{Br}$ J Dermatol. 2016;175(4):687-695.

40. Higaki S, Morohashi M, Yamagishi T, Hasegawa Y. Comparative study of staphylococci from the skin of atopic dermatitis patients and from healthy subjects. Int J Dermatol. 1999;38(4):265-269.

41. Hon KL, Tsang YC, Pong NH, Leung TF, Ip M. Exploring Staphylococcus epidermidis in atopic eczema: friend or foe? Clin Exp Dermatol. 2016;41(6):659-663.

42. Sugimoto S, Iwamoto T, Takada K, et al. Staphylococcus epidermidis Esp degrades specific proteins associated with Staphylococcus aureus biofilm formation and host-pathogen interaction. J Bacteriol. 2013;195(8):1645-1655.

43. Otto M. Staphylococcus colonization of the skin and antimicrobial peptides. Expert Rev Dermatol. 2010;5(2):183-195.

44. Cogen AL, Yamasaki K, Sanchez KM, et al. Selective antimicrobial action is provided by phenol-soluble modulins derived from Staphylococcus epidermidis, a normal resident of the skin. J Invest Dermatol. 2010;130(1):192-200.

45. Callewaert C, Nakatsuji T, Knight R, et al. IL-4Ralpha blockade by dupilumab decreases Staphylococcus aureus colonization and increases microbial diversity in atopic dermatitis. J Invest Dermatol. 2019. In Press.

\section{SUPPORTING INFORMATION}

Additional supporting information may be found online in the Supporting Information section at the end of the article.

How to cite this article: van Mierlo M, Totté J, Fieten KB, et al. The influence of treatment in alpine and moderate maritime climate on the composition of the skin microbiome in patients with difficult to treat atopic dermatitis. Clin Exp Allergy. 2019;49:1437-1445. https://doi.org/10.1111/cea.13492 